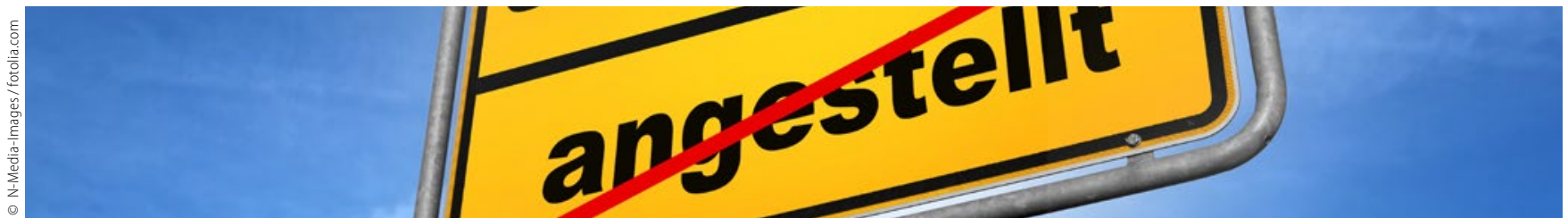

Der Weg in die Selbstständigkeit ist machbar

\title{
Vorbereitungszeit zu Ende - und jetzt?
}

\author{
Für die meisten der freiberuflich tätigen Zahnärzte war das noch keine Frage: Die Selbstständigkeit \\ war nahezu alternativlos. Doch heute gibt es etliche Alternativen. Und so stellt sich spätestens \\ während der Vorbereitungszeit jeder Assistent die Frage, wie es weitergehen soll.
}

\section{Da gibt es verschiedene Möglichkeiten:}

- (Zunächst) weiter als angestellter Zahnarzt tätig sein in einer Einzelpraxis, Berufsausübungsgemeinschaft (BAG) oder einer Klinik?

- Eine eigene Praxis gründen?

- Die Praxis eines Kollegen übernehmen?

- In eine BAG einsteigen?

Die erste Frage, die in diesem Zusammenhang zu beantworten ist, ist grundsätzlicher Natur: Angestellt bleiben oder selbstständig tätig werden? Die Antwort auf diese Frage fällt nicht leicht, gibt es doch gute Argumente für beide Modelle. Für die Selbstständigkeit sprechen zwei Motive: das Motiv der Autonomie und das Statusmotiv. Nur in der Selbstständigkeit kann der Zahnarzt

- bei den gesetzlichen und vom Markt vorgegebenen Möglichkeiten über die Art und die Qualität seines Leistungsangebots, seine Arbeitszeiten, die Menschen, mit denen er arbeiten möchte und vieles mehr weitgehend unabhängig entscheiden;

- die Früchte seiner Arbeit selbst ernten und

- in seinem Umfeld den hohen Status des Freiberuflers erwerben.

Dinge, die jeder Zahnarzt wertschätzt, wenn da nicht die damit verbundenen Verpflichtungen wären:

\section{Die Praxisfinanzierung}

Grundsätzlich ist es möglich, Investitionen durchzurechnen, um so zu prüfen, ob sich ein Investment lohnt. Wer sich das allein nicht zutraut, sollte eine Bank hinzuziehen. Sie wird gerne die erforderlichen Modellrechnungen übernehmen. Angesichts der aktuell günstigen Zinssituation sind die finanziellen Risiken zu vernachlässigen, wenn die Zinsen langfristig gesichert werden.

\section{Die Notwendigkeit, sich im Wettbewerb durchzusetzen}

Mit einem klaren Praxiskonzept vor Augen fällt es leicht, sich von Kollegen vor Ort abzusetzen. Je mehr Erfahrungen in Praktika während der Studienzeit, in der Assistenzzeit oder auch im Ausland gesammelt wurden, desto klarer wird das Bild von der idealen Praxis. Man weiß ein wenig mehr, was sich hinsichtlich der Praxisausstattung und der Praxisführung bewährt hat und was nicht. Vor allem aber wird der Zahnarzt mit jeder neuen Erfahrung sicherer in seinem Urteil.

\section{Die vielfältigen administrativen Aufgaben}

Auch das ist fester Bestandteil einer Selbstständigkeit, die $\mathrm{Pa}-$ lette der administrativen Aufgaben reicht von der Abrechnung über Dokumentation, EDV, Hygienemanagement, Organisation, Personalmanagement, QM bis Zeitmanagement und vieles andere mehr.

Auch diese vielfältigen Aufgaben lassen sich nach und nach bewältigen. Das notwendige Fachwissen gibt es zum Beispiel in den Seminaren, die über den FVDZ und seinen Kooperationspartner ZA angeboten werden. Wichtig ist es auch, keine Angst vor Fehlern zu haben. Sie sind der Schlüssel zum Erfolg. Sie helfen, die Dinge künftig besser zu machen.

Bei näherer Betrachtung sind die von jungen Zahnärzten wahrgenommenen Risiken also beherrschbar. Daher gilt das Fazit: Es lohnt sich auch heute noch, den Schritt in die Selbstständigkeit zu gehen.

\begin{tabular}{ll} 
T1 Praxisfinanzierung & \\
\hline & 2014 in $€$ \\
Übernahme einer Einzelpraxis & 323.000 \\
Neugründung einer Einzel praxis & 422.000 \\
Übernahme einer BAG & 262.000 \\
Neugründung einer BAG & 312.000 \\
Beitritt in eine BAG & 281.000 \\
Quelle: IDZ-Information 3/15 & \\
\hline
\end{tabular}

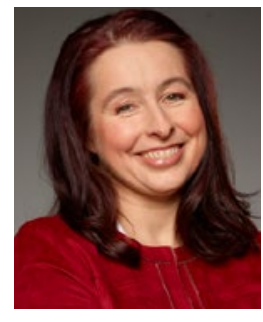

Dr. Susanne Woitzik swoitzik@za-eg.de 